Journal of Applied Mathematics and Stochastic Analysis, 16:2 (2003), 163-170.

Printed in the USA (C)2003 by North Atlantic Science Publishing Company

\title{
EXISTENCE OF SOLUTIONS OF SOBOLEV-TYPE SEMILINEAR MIXED INTEGRODIFFERENTIAL INCLUSIONS IN BANACH SPACES
}

\author{
M. KANAKARAJ and K. BALACHANDRAN \\ Bharathiar University, Department of Mathematics \\ Coimbatore-641046, India \\ E-mail: balachandran_k@lycos.com
}

(Received January 2002; Revised March 2003)

\begin{abstract}
The existence of mild solutions of Sobolev-type semilinear mixed integrodifferential inclusions in Banach spaces is proved using a fixed point theorem for multivalued maps on locally convex topological spaces.

Keywords: Integrodifferential Inclusion, Convex Multivalued Map, Fixed Point Theorem.
\end{abstract}

AMS (MOS) Subject Classification: 34A60, 34G20, 45J05.

\section{Introduction}

The problem of proving the existence of mild solutions for differential and integrodifferential equations in abstract spaces has been studied by several authors $[2,4,11,12,13]$. Balachandran and Uchiyama [3] established the existence of solutions of nonlinear integrodifferential equations of Sobolev type with nonlocal conditions in Banach spaces. Benchohra [6] studied the existence of mild solutions on infinite intervals for a class of differential inclusions in Banach spaces. For the existence results of differential inclusions on compact intervals, one can refer to the papers of Avgerinos and Papageorgiou [1], and Papageorgiou [14, 15]. Benchohra and Ntouyas [7] discussed the existence results for first order integrodifferential inclusions of the form

$$
\begin{gathered}
\frac{d y}{d t}-A y \in F\left(t, \int_{0}^{t} k(t, s, y) d s\right) \quad t \in I=[0, \infty), \\
y(0)=y_{0} .
\end{gathered}
$$

In this paper, we consider the Sobolev-type semilinear mixed integrodifferential inclusion of the type

$$
(E u(t))^{\prime}+A u \in G\left(t, u, \int_{0}^{t} k(t, s, u) d s, \int_{0}^{a} b(t, s, u) d s\right) \quad t \in I=[0, \infty),(1.1
$$




$$
u(0)=u_{0},
$$

where $G: I \times X \times X \times X \rightarrow 2^{Y}$ is a bounded, closed, convex, multivalued map $k: \Delta \times X \rightarrow X, \quad b: \Delta \times X \rightarrow X$, where $\Delta=\{(t, s) \in I \times I ; t \geq s\}, u_{0} \in X, a$ is a real constant, $X, Y$ are real Banach spaces with norms $\|$.$\| and |.|, respectively. Our method$ is to reduce the problem (1.1) to a fixed point problem of a suitable multivalued map in the Frechet space $C(I, X)$ and we make use of a fixed point theorem due to Ma [10] for multivalued maps in locally convex topological spaces.

\section{Preliminaries}

In this section we introduce the notations, definitions and preliminary facts from multivalued analysis which are used in this paper. $I_{m}$ is the compact interval $[0, m](m \in N)$. $C(I, X)$ is the linear metric Frechet space of continuous functions from $I$ into $X$ with the metric

$$
d(u, z)=\sum_{m=0}^{\infty} \frac{2^{-m}\|u-z\|_{m}}{1+\|u-z\|_{m}} \text { for each } u, z \in C(I, X),
$$

where $\|u\|_{m}=\sup \left\{\|u(t)\|: t \in I_{m}\right\} . B(X)$ denotes the Banach space of bounded linear operators from $X$ into $X$. A measurable function $u: I \rightarrow X$ is Bochner integrable if and only if $|u|$ is Lebesgue integrable. Let $L^{1}(I, X)$ denote the Banach space of continuous functions $u: I \rightarrow X$ which are Bochner integrable normed by

$$
\|u\|_{L^{1}}=\int_{0}^{\infty}\|u(t)\| d t
$$

and $U_{r}$ is a neighbourhood of 0 in $C(I, X)$ defined by

$$
U_{r}=\left\{u \in C(I, X):\|u\|_{m} \leq r\right\}
$$

for each $m \in N$. The convergence in $C(I, X)$ is the uniform convergence on compact intervals, that is, $u_{j} \rightarrow u$ in $C(I, X)$ if and only if for each $m \in N,\left\|u_{j}-u\right\|_{m} \rightarrow 0$ in $C\left(I_{m}, X\right)$ as $j \rightarrow \infty$. BCC $(X)$ denotes the set of all nonempty bounded, closed, and convex subsets of $X$.

A multivalued map $G: X \rightarrow 2^{X}$ is convex(closed) valued if $G(x)$ is convex(closed) for all $x \in X$. $G$ is bounded on bounded sets if $G(B)=\bigcup_{x \in B} G(x)$ is bounded in $X$ for any bounded set $B$ of $X$ (that is, $\sup _{x \in B}\{\sup \{\|u\|: u \in G(x)\}\}<\infty$ ). $G$ is called upper semi continuous on $\mathrm{X}$ if for each $x_{0} \in X$ the set $G\left(x_{0}\right)$ is a nonempty, closed subset of $X$, and if for each open subset $B$ of $X$ containing $G\left(x_{0}\right)$, there exists an open neighbourhood $A$ of $x_{0}$ such that $G(A) \subseteq B$. $G$ is said to be completely continuous if $G(B)$ is relatively compact for every bounded subset $B \subseteq X$. If the multivalued map $G$ is completely continuous with nonempty compact values, then $G$ is upper semicontinuous if and only if $G$ has a closed graph (that is, $x_{n} \rightarrow x_{0}, u_{n} \rightarrow u_{0}, u_{n} \in G x_{n}$ imply $u_{0} \in G x_{0}$ ).

We assume the following conditions:

(i) The operator $A: D(A) \subset X \rightarrow Y$ and $E: D(E) \subset X \rightarrow Y$ satisfy the following conditions 
$\left[C_{1}\right] A$ and $E$ are closed linear operators.

$\left[C_{2}\right] D(E) \subset D(A)$ and $E$ is bijective.

$\left[C_{3}\right] E^{-1}: Y \rightarrow D(E)$ is continuous.

$\left[C_{4}\right]$ The resolvent $R\left(\lambda,-A E^{-1}\right)$ is a compact operator for some $\lambda \in \rho\left(-A E^{-1}\right)$ and resolvent set of $-A E^{-1}$.

Conditions $\left[C_{1}\right],\left[C_{2}\right]$, and the closed graph theorem imply the boundedness of the linear operator $A E^{-1}: Y \rightarrow Y$.

(ii) $G: I \times X \times X \times X \rightarrow B C C(Y)$ is measurable with respect to $\mathrm{t}$ for each $u \in X$, upper semi continuous with respect to $\mathrm{u}$ for each $t \in I$, and for each $u \in C(I, X)$ the set

$$
S_{G, u}=\left\{g \in L^{1}(I ; R): g(t) \in G\left(t, u, \int_{0}^{t} k(t, s, u) d s, \int_{0}^{a} b(t, s, u) d s\right)\right.
$$

for a.e $t \in I\}$ is nonempty.

(iii) There exist functions $p(t), q(t) \in C(I ; R)$ such that

$$
\left|\int_{0}^{t} k(t, s, u) d s\right| \leq p(t)\|u\| \text { and }\left|\int_{0}^{a} b(t, s, u) d s\right| \leq q(t)\|u\| \text { for a.e } t, s \in I, u \in X .
$$

(iv) There exists a function $\alpha(t) \in L^{1}\left(I ; R^{+}\right)$such that

$$
\|G(t, u, v, w)\| \leq \alpha(t) \Omega(\|u\|+\|v\|+\|w\|)
$$

for a.e $t \in I, u \in X$, where $\Omega: R_{+} \rightarrow(0, \infty)$ is continuous increasing function satisfying $\Omega(p(t) x+q(t) y) \leq p(t) \Omega(x)+q(t) \Omega(y)$ and

$$
M \int_{0}^{m} \alpha(s)(1+p(s)+q(s)) d s<\int_{c}^{\infty} \frac{d u}{\Omega(u)}
$$

for each $m \in N$, where $c=\left\|E^{-1}\right\| M\left|E u_{0}\right|$ and $M=\max \{\|T(t)\| ; t \in I\}$.

(v) For each neighbourhood $U_{r}$ of $0, u \in U_{r}$ and $t \in I$, the set

$$
\left\{E^{-1} T(t) E u_{0}+\int_{0}^{t} E^{-1} T(t-s) g(s) d s, g \in S_{G, u}\right\}
$$

is relatively compact.

Definition 2.1: A continuous function $u(t)$ of the integral inclusion

$$
u(t) \in E^{-1} T(t) E u_{0}+\int_{0}^{t} E^{-1} T(t-s) G\left(s, u, \int_{0}^{s} k(s, \tau, u(\tau)) d \tau, \int_{0}^{a} b(s, \tau, u(\tau)) d \tau\right) d s
$$

is called a mild solution of (1.1) on $I$.

Lemma 2.1: [9]. Let I be a compact real interval and let $X$ be a Banach space. Let $G$ be a multivalued map satisfying $(i)$ and let $\Gamma$ be a linear continuous mapping from $L^{1}(I, X)$ to $C(I, X)$. Then the operator

$$
\Gamma \circ S_{G}: C(I, X) \rightarrow X, \quad\left(\Gamma \circ S_{G}\right)(y)=\Gamma\left(S_{G, y}\right)
$$


is a closed graph operator in $C(I, X) \times C(I, X)$.

Lemma 2.2: [10]. Let $X$ be a locally convex space. Let $N: X \rightarrow X$ be a compact, convex valued, upper semicontinuous, multivalued map such that there exists a closed neighbourhood $U_{r}$ of 0 for which $N\left(U_{r}\right)$ is a relatively compact set for each $r \in N$. If the set $\zeta=\{y \in X: \lambda y \in N(y)\}$ for some $\lambda>1$ is bounded, then $N$ has a fixed point.

Remark: [9]. If $\operatorname{dim} X<\infty$ and $I$ is a compact real interval, then for each $u \in$ $C(I, X), \quad S_{G, u}$ is nonempty.

Lemma 2.3: [16]. Let $S(t)$ be a uniformly continuous semigroup and let $A$ be its infinitesimal generator. If the resolvent set $R(\lambda: A)$ of $A$ is compact for every $\lambda \in \rho(A)$, then $S(t)$ is a compact semigroup.

From the above fact, $-A E^{-1}$ generates a compact semigroup $T(t)$ in $Y$. Thus, $\max _{t \in I}|T(t)|$ is finite and so denote $M=\max _{t \in I}|T(t)|$.

\section{Main Result}

Theorem 3.1: If the assumptions $(i)-(v)$ are satisfied, then the initial value problem (1.1) has at least one mild solution on $I$.

Proof: A solution to (1.1) is a fixed point for the multivalued map $N: C(I, X) \rightarrow 2^{C(I, X)}$ defined by

$$
N(u)=\left\{h \in C(I, X): h(t)=E^{-1} T(t) E u_{0}+\int_{0}^{t} E^{-1} T(t-s) g(s) d s, g \in S_{G, u}\right\},
$$

where

$$
S_{G, u}=\left\{g \in L^{1}(I, X): g(t) \in G\left(t, u, \int_{0}^{t} k(t, s, u(s)) d s, \int_{0}^{a} b(t, s, u(s)) d s\right)\right.
$$

for a.e $t \in I\}$.

First we shall prove $N(u)$ is convex for each $u \in C(I, X)$. Let $h_{1}, h_{2} \in N(u)$, then there exist $g_{1}, g_{2} \in S_{G, u}$ such that

$$
h_{i}(t)=E^{-1} T(t) E u_{0}+\int_{0}^{t} E^{-1} T(t-s) g_{i}(s) d s, i=1,2, t \in I
$$

Let $0 \leq k_{1} \leq 1$, then for each $t \in I$ we have

$$
\left(k_{1} h_{1}+\left(1-k_{1}\right) h_{2}\right) t=E^{-1} T(t) E u_{0}+\int_{0}^{t} E^{-1} T(t-s)\left(k_{1} g_{1}(s)+\left(1-k_{1}\right) g_{2}(s)\right) d s .
$$

Since $S_{G, u}$ is convex, thus $k h_{1}+(1-k) h_{2} \in N(u)$. Hence, $N(u)$ is convex for each $u \in C(I, X)$.

Let $U_{r}=\{u \in C(I, X) ;\|u\| \leq r\}$ be a neighbourhood of 0 in $C(I, X)$ and $u \in U_{r}$. Then for each $h \in N(u)$ there exists $g \in S_{G, u}$ such that for $t \in I$, we have

$$
\begin{aligned}
\|h(t)\| & \leq\left\|E^{-1}\right\|\|T(t)\|\left|E u_{0}\right|+\int_{0}^{t}\left\|E^{-1}\right\|\|T(t-s)\|\|g(s)\| d s \\
& \leq\left\|E^{-1}\right\| M\left|E u_{0}\right|+\left\|E^{-1}\right\| M \int_{0}^{t} \alpha(s) \Omega(\|u\|+p(t)\|u\|+q(t)\|u\|) d s
\end{aligned}
$$




$$
\begin{aligned}
& \leq\left\|E^{-1}\right\| M\left|E u_{0}\right|+\left\|E^{-1}\right\| M \int_{0}^{t} \alpha(s)(\Omega(\|u\|)+p(t) \Omega(\|u\|)+q(t) \Omega(\|u\|)) d s \\
& \leq\left\|E^{-1}\right\| M\left|E u_{0}\right|+\left\|E^{-1}\right\| M \int_{0}^{t} \alpha(s)(1+p(s)+q(s)) \Omega(\|u\|) d s \\
& \leq\left\|E^{-1}\right\| M\left|E u_{0}\right|+\left\|E^{-1}\right\| M\|\alpha\|_{L^{1}\left(I_{m}\right)}\|(1+p(s)+q(s))\| \sup _{u \in U_{r}} \Omega(\|u\|)
\end{aligned}
$$

Hence, $N\left(U_{r}\right)$ is bounded in $C(I, X)$ for each $r \in N$. Next we shall prove $N\left(U_{r}\right)$ is an equicontinuous set in $C(I, X)$ for each $r \in N$. Let $t_{1}, t_{2} \in I_{m}$ with $t_{1}<t_{2}$. Then for all $h \in N(u)$ with $u \in U_{r}$, we have

$$
\begin{aligned}
\left\|h\left(t_{1}\right)-h\left(t_{2}\right)\right\| \leq & \left\|E^{-1}\right\|\left\|\left(T\left(t_{2}\right)-T\left(t_{1}\right)\right) E u_{0}\right\| \\
& +\left\|E^{-1}\right\|\left\|\int_{0}^{t_{2}}\left(T\left(t_{2}-s\right)-T\left(t_{1}-s\right)\right) g(u) d s\right\| \\
& +\left\|E^{-1}\right\|\left\|\int_{t_{1}}^{t_{2}} T\left(t_{1}-s\right) g(u) d s\right\| \\
\leq & \left\|E^{-1}\right\|\left\|\left(T\left(t_{2}\right)-T\left(t_{1}\right)\right) E u_{0}\right\| \\
& +\left\|E^{-1}\right\|\left\|\int_{0}^{t_{2}}\left(T\left(t_{2}-s\right)-T\left(t_{1}-s\right)\right) g(u) d s\right\| \\
& +M\left(t_{2}-t_{1}\right)\left\|E^{-1}\right\| \int_{0}^{m}\|g(s)\| d s .
\end{aligned}
$$

Hence, by the Ascoli-Arzela Theorem, we conclude that $N: C(I, X) \rightarrow 2^{C(I, X)}$ is a completely continuous multivalued map. Next we shall prove that $N$ has a closed graph. Let $u_{n} \rightarrow u_{*}, h_{n} \in N\left(u_{n}\right)$ and $h_{n} \rightarrow h_{0}$, then we shall prove that $h_{0} \in N\left(u_{*}\right)$. Here, $h_{n} \in N\left(u_{n}\right)$ means that there exists $g_{n} \in S_{G, u_{n}}$ such that

$$
h_{n}(t)=E^{-1} T(t) E u_{0}+\int_{0}^{t} E^{-1} T(t-s) g_{n}(s) d s, \quad t \in I .
$$

We must also prove that there exists $g_{0} \in S_{G, u}$ such that

$$
h_{0}(t)=E^{-1} T(t) E u_{0}+\int_{0}^{t} E^{-1} T(t-s) g_{0}(s) d s, \quad t \in J .
$$

To prove the above, we use the fact that $h_{n} \rightarrow h_{0}$; and $h_{n}-E^{-1} T(t) E u_{0} \in \Gamma\left(S_{G, u}\right)$, where

$$
(\Gamma g)(t)=\int_{0}^{t} E^{-1} T(t-s) g(s) d s, \quad t \in I .
$$

Consider the functions $u_{n}, h_{n}-E^{-1} T(t) E u_{0}$ and $g_{n}$ defined on the interval $[k, k+1]$ for any $k \in N \cup\{0\}$. Then using Lemma 2.1, we can conclude (3.1) is true on the compact interval $[k, k+1]$. That is,

$$
\left[h_{0}(t)\right]_{[k, k+1]}=E^{-1} T(t) E u_{0}+\int_{0}^{t} E^{-1} T(t-s) g_{0}^{k}(s) d s
$$

for a suitable $L^{1}$-selection $g_{0}^{k}$ of $G\left(t, u, \int_{0}^{t} k(t, s, u) d s, \int_{0}^{T} b(t, s, u) d s\right)$ on the interval $[k, k+1]$. Let $g_{0}(t)=g_{0}^{k}(t)$ for $t \in[k, k+1)$. Then $g_{0}$ is an $L^{1}$-selection and (3.1) 
will satisfied. Clearly we have $\left\|\left(h_{n}-E^{-1} T(t) E u_{0}\right)-\left(h_{0}-E^{-1} T(t) E u_{0}\right)\right\|_{\infty} \rightarrow 0$ as $n \rightarrow \infty$. Consider for all $k \in N \cup\{0\}$, the mapping

$$
\begin{gathered}
S_{G}^{k}: C([k, k+1], X) \rightarrow L^{1}([k, k+1], X), \\
y \rightarrow S_{G, y}^{k}=\left\{g \in L^{1}([k, k+1], X): g(t) \in G\left(t, u, \int_{0}^{t} k(t, s, u) d s, \int_{0}^{a} b(t, s, u) d s\right)\right.
\end{gathered}
$$

for a.e $t \in[k, k+1]\}$.

Now we consider the linear continuous operators

$$
\begin{gathered}
\Gamma_{k}: L^{1}([k, k+1], X) \rightarrow C([k, k+1], X), \\
g \rightarrow \Gamma_{k}(g)(t)=\int_{0}^{t} E^{-1} T(t-s) g(s) d s .
\end{gathered}
$$

From Lemma 2.1 it follows that $\Gamma_{k} \circ S_{G}^{k}$ is a closed graph operator for all $k \in N \cup\{0\}$. Moreover, we have

$$
\left.\left(h_{n}(t)-E^{-1} T(t) E u_{0}\right)\right|_{[k, k+1]} \in \Gamma_{k}\left(S_{G, u_{n}}^{k}\right)
$$

and $u_{n} \rightarrow u_{*}$. From Lemma 2.1, we have $\left.\left(h_{0}(t)-E^{-1} T(t) E u_{0}\right)\right|_{[k, k+1]} \in \Gamma_{k}\left(S_{G, u_{*}}^{k}\right)$,

$$
\left.\left(h_{0}(t)-E^{-1} T(t) E u_{0}\right)\right|_{[k, k+1]}=\int_{0}^{t} E^{-1} T(t-s) g_{0}^{k}(s) d s \text { for some } g_{0}^{k} \in S_{G, u_{*}}^{k} .
$$

Hence, the function $g_{0}$ defined on $I$ by $g_{0}(t)=g_{0}^{k}(t)$ for $t \in[k, k+1]$ is in $S_{G, u_{*}}$. Therefore, $N\left(U_{r}\right)$ is relatively compact for each $r \in N$ where $N$ is upper semicontinuous with convex closed values. Finally we prove the set $\zeta=\{u \in C(I, X) ; \lambda u \in N u\}$, for some $\lambda>1$, is bounded.

Let $\lambda u=N u$ for some $\lambda>1$. Then there exists $g \in S_{G, u}$ such that

$$
\begin{array}{r}
u(t)=\lambda^{-1} E^{-1} T(t) E u_{0}+\lambda^{-1} \int_{0}^{t} E^{-1} T(t-s) g(s) d s, t \in I, \\
\|u(t)\| \leq\left\|E^{-1}\right\| M\left|E u_{0}\right|+\left\|E^{-1}\right\| M \int_{0}^{t} \alpha(s)(1+p(s)+q(s)) \Omega(\|u\|) d s .
\end{array}
$$

Let $v(t)=\left\|E^{-1}|| M\left|E u_{0}\right|+\right\| E^{-1} \| M \int_{0}^{t} \alpha(s)(1+p(s)+q(s)) \Omega(\|u\|) d s$. Then we have $v(0)=\left\|E^{-1}\right\| M\left\|E u_{0}\right\|=c$ and $\|u(t)\| \leq v(t), t \in I_{m}$. Using the increasing character of $\Omega$ we get

$$
v^{\prime}(t) \leq\left\|E^{-1}\right\| M \alpha(t)(1+p(t)+q(t)) \Omega(v(t)), \quad t \in I_{m} .
$$

The above proves that for each $t \in I_{m}$,

$$
\int_{v(0)}^{v(t)} \frac{d u}{\Omega(u)} \leq\left\|E^{-1}\right\| M \int_{0}^{m} \alpha(s)(1+p(s)+q(s)) d s<\int_{0}^{\infty} \frac{d u}{\Omega(u)} .
$$

The above inequality implies that there exists a constant $M_{0}$ such that $v(t) \leq M_{0}, t \in$ $I_{m}$, and hence that $\|u\|_{\infty} \leq M_{0}$ where $M_{0}$ depends on $\mathrm{m}$ and on the functions $\alpha, p, \Omega$. Hence, $\zeta$ is bounded. Thus by Lemma $2.2, N$ has a fixed point that is a mild solution of (1.1). 


\section{Nonlocal Initial Conditions}

Several authors have studied the nonlocal Cauchy problem in abstract spaces $[2,3,4$, $11,12,13]$. The importance of nonlocal conditions is discussed in $[4,5]$. In this section we consider a first order Sobolev-type, semilinear, mixed, integrodifferential inclusion (1.1) with the nonlocal initial condition

$$
u(0)+f(u)=u_{0}
$$

In addition to the five assumptions in Section 2, we also assume the following.

(vi) $f: C(I, X) \rightarrow X$ is a continuous function, and there exists a constant $L>0$ such that $\|f(u)\| \leq L$ for each $u \in X$.

(vii) $\left\|E^{-1}\right\| M \int_{0}^{m} \alpha(s)(1+p(s)+q(s)) d s<\int_{c_{1}}^{\infty} \frac{d u}{\Omega(u)}$ where $c_{1}=\| E^{-1}|| M\left|E u_{0}\right|+$ $L \| E^{-1}|| M\left|E u_{0}\right|$.

(viii) For each neighbourhood $U_{r}$ of $0, u \in U_{r}$ and $t \in I$, the set $\left\{E^{-1} T(t) E u_{0}-\right.$ $\left.E^{-1} T(t) E f(u)+\int_{0}^{t} E^{-1} T(t-s) g(s) d s, g \in S_{G, u}\right\}$ is relatively compact.

Definition 4.1: A continuous function $u(t)$ of the integral inclusion

$$
\begin{aligned}
u(t) & \in E^{-1} T(t) E u_{0}-E^{-1} T(t) E f(u) \\
& +\int_{0}^{t} E^{-1} T(t-s) G\left(s, u, \int_{0}^{s} k(s, \tau, u(\tau)) d \tau, \int_{0}^{a} b(s, \tau, u(\tau)) d \tau\right) d s
\end{aligned}
$$

is called a mild solution of (1.1)-(4.1) on I.

Theorem 4.1: If the assumptions $(i)-(i i i)$, (vi)-(viii) are satisfied, then the nonlocal initial value problem (1.1)-(4.1) has at least one mild solution on I.

The proof of Theorem 4.1 is similar to Theorem 3.1 and hence, is omitted.

\section{References}

[1] Avgerinos, E.P. and Papageorgiou, N.S., On quasilinear evolution inclusions, Glas. Mat.Ser. III 28:48 (1993), 35-52.

[2] Balachandran, K. and Chandrasekaran, M., The nonlocal Cauchy problem for semilinear integrodifferential equations with deviating argument, Proc. Edinburgh Math.Soc. 44 (2001), 63-70.

[3] Balachandran, K. and Uchiyama, K., Existence of solutions of nonlinear integrodifferential equations of Sobolev type with nonlocal condition in Banach spaces, Proc. Indian Acad. Sci. Math. Sci. 110 (2000), 225-232.

[4] Balachandran, K. and Uchiyama, K., Existence of solutions of quasilinear integrodifferential equations with nonlocal condition, Tokyo J. Math. 23 (2000), 203-210.

[5] Byszewski, L., Theorems about the existence and uniqueness of solutions of a semilinear evolution nonlocal Cauchy problem, J. Math. Anal. Appl. 162 (1992), 495-505.

[6] Benchohra, M., Existence of mild solutions on infinite intervals to first order initial value problems for a class of differential inclusions in Banach spaces, Disc. Math. and Differ. Inclus. 19 (1999), 111-121. 
[7] Benchohra, M. and Ntouyas, S.K., An Existence result on noncompact intervals to first order integrodifferential inclusions in Banach spaces, Libertas Mathematica 20 (2000), $71-78$.

[8] Corduneanu, C., Integral Equations and Applications, Cambridge Univ.Press, New York 1990.

[9] Lasota, A. and Opial, Z., An application of the Kakutani-Ky-Fan theorem in the theory of ordinary differential equations, Bull. Acad. Polon. Sci. Ser. Sci. Math. Astronom. Phys. 13 (1965), 781-786.

[10] Ma, T.W., Topological degrees for set-valued compact vector fields in locally convex spaces, Dissertationess Math. 92 (1972), 1-43.

[11] Ntouyas, S.K. and Tsamatos, P.Ch., Global existence for second order semilinear ordinary and delay integrodifferential equations with nonlocal conditions, Applic. Anal. 67 (1997), $245-257$.

[12] Ntouyas, S.K. and Tsamatos, P.Ch., Global existence for semilinear evolution integrodifferential equations with delay and nonlocal conditions, Applic. Anal. 64 (1997), 99-105.

[13] Ntouyas, S.K., Global existence results for certain second order delay integrodifferential equations with nonlocal conditions, Dynam. Systems Appl. 7 (1998), 415-426.

[14] Papageorgiou, N.S., Mild solutions of semilinear evolution inclusions, Indian J. Pure Appl. Math. 26 (1995), 189-216.

[15] Papageorgiou, N.S., Boundary value problems for evolution inclusions, Comment. Math. Univ. Carol. 29 (1988), 355-363.

[16] Pazy, A., Semigroups of Linear Operators and Applications to Partial Differential Equations, Springer-Verlag, New York 1983. 


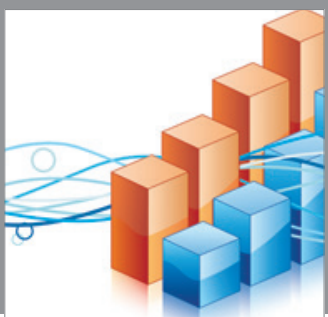

Advances in

Operations Research

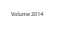

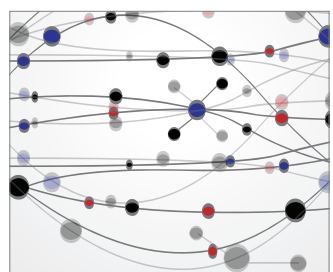

\section{The Scientific} World Journal
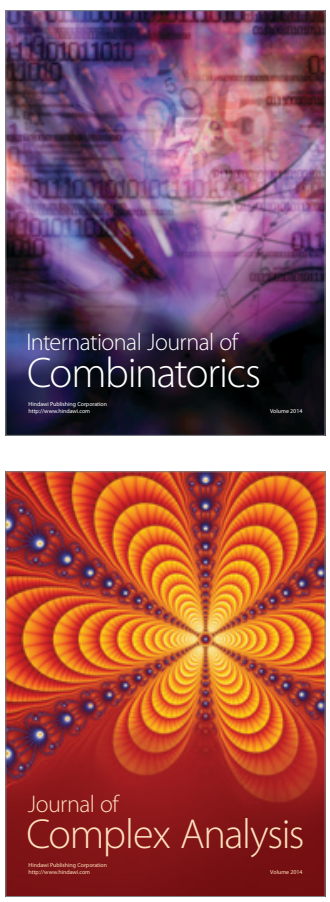

International Journal of

Mathematics and

Mathematical

Sciences
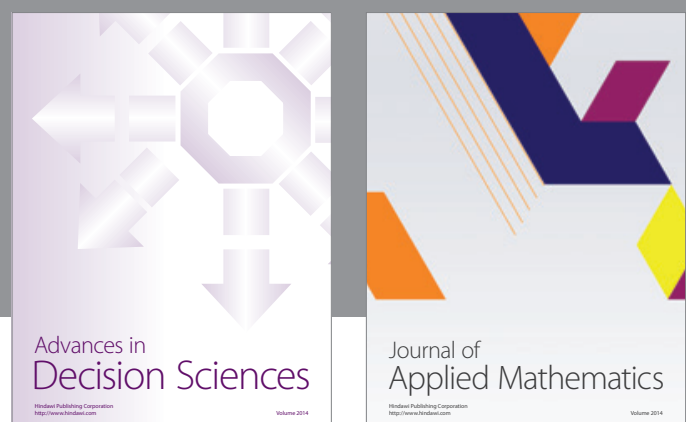

Journal of

Applied Mathematics
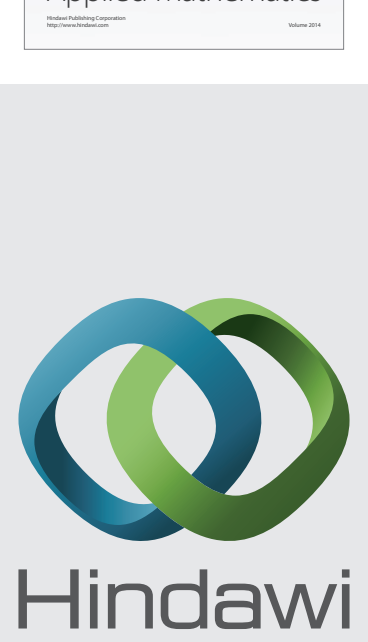

Submit your manuscripts at http://www.hindawi.com
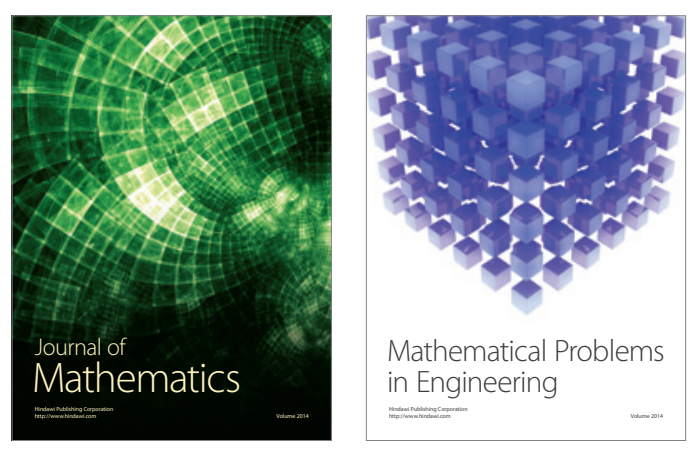

Mathematical Problems in Engineering
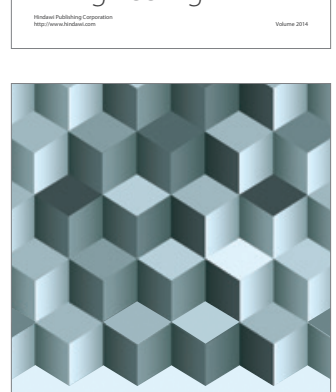

Journal of

Function Spaces
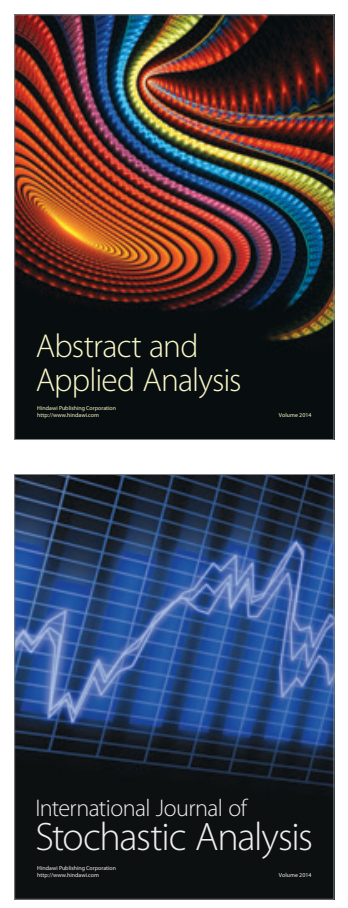

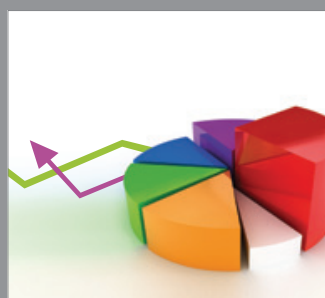

ournal of

Probability and Statistics

Promensencen
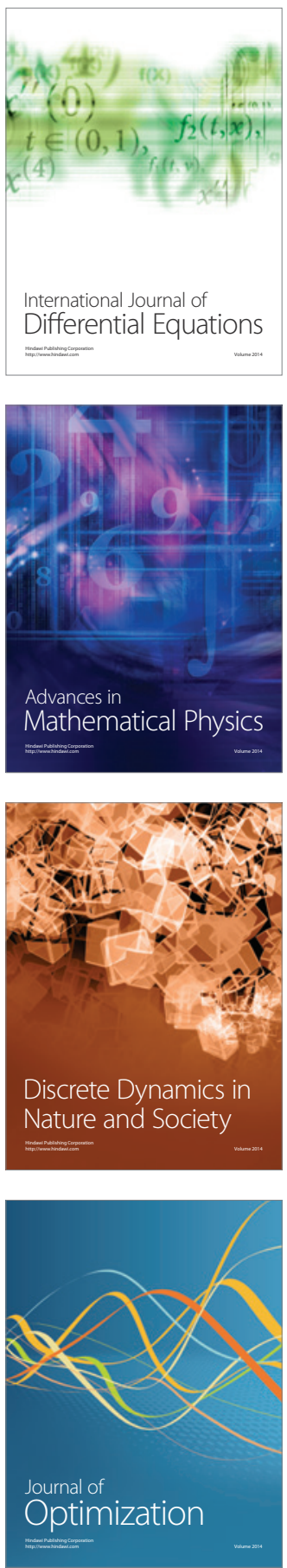